\title{
PLANETARY NEBULAE WITH THE STRONG [NII] EMISSION LINES
}

\author{
L. N. KONDRATJEVA \\ Astrophysical Institute of Kazakh Academy of Sciences, Kamenskoje Plato, \\ 480068 Alma-Ata, Kazakhstan
}

\begin{abstract}
The sample of planetaries with the strong [NII] emission lines are analysed. All available parameters are discussed. The enrichments of all elements and the tendency to rather low surface brightness of ne bulae in $\mathrm{H} \beta$ flux are remarked. The deficiency of hydrogen in envelopes is proposed as the possible reason of observational spectra.
\end{abstract}

\section{INTRODUCTION}

The spectra of planetary nebulae with the strongest [NII] emission lines can't be represented with traditional photoionization models because the computed intensities are lower than observational values. The only way out is to change the abundances in order to come to an agreement with observations. The spectra in which the strongest [NII] lines coexist with HeII emission are of special interest. Usually such objects are considered to be massive ionization- bounded nebulae of the Population I type. The aim of our research was to analyse the physical parameters of such object in order to single out the features peculiar just to them.

\section{RESULTS}

We have compiled a list of about 300 planetaries studied in the wide wavelength region and then the objects with $\mathrm{I}([\mathrm{NII}] \lambda 6548+\lambda 6583) / \mathrm{I}(\mathrm{H} \alpha) \geq 1.0$ and $\mathrm{I}(\mathrm{HeII} \lambda 4686) /$ $\mathrm{I}(\mathrm{H} \beta)>0.2$. To avoid the $\mathrm{N}$ abundance anomaly we have choosen only those objects with $[\mathrm{NII}] /[\mathrm{SII}]$ ratio close to the average value of about 10 .Our sample consists of 20 planetaries. They have quite normal $\mathrm{T}_{e}$ and $\mathrm{N}_{e}$ and $\mathrm{T}_{e f f}>60000 \mathrm{~K}$. All available abundances of $\mathrm{HE}, \mathrm{N}, \mathrm{NE}, \mathrm{O}, \mathrm{S}, \mathrm{Ar}$ are significantly higher than the average values adopted for planetaries. The derived surface brightnesses are rather low indicating the small nebular masses or the low stellar luminosities.

\section{DISCUSSION}

All observational and derived features may be explained if we suppose the defiency of hydrogen in selected envelopes. If so the nebular masses must be reduced as a factor of 2-4 in dependence on the hydrogen deficit degree. The stellar luminosities must be low enough to adjust the small nebular masses with the large optical depths. Thus the central stars may be expected to take place in the lower part of H-R diagram for nuclei, somewhere close to white dwarfs, and our sample is appeared to be rather old planetaries. The extreme cases of hydrogen deficit are observed in A30 and A78, their secondary envelopes are almost devoid of hydrogen. Probably the nebulae with selected spectra are the results of the second throw out as well. 